\title{
Integration of HIV-I Caused STAT3-Associated B Cell Lymphoma in an AIDS Patient
}

\author{
Harutaka Katano* ${ }^{*}$, Yuko Sato1, Satomi Hoshino², Shigeo Mori³, \\ Tetsutaro Sata1, Micheal D Weiden ${ }^{2}$ and Yoshihiko Hoshino ${ }^{2}$
}

\author{
Address: ${ }^{1}$ Department of Pathology, National Institute of Infectious Diseases, Tokyo 162-8640, Japan, ${ }^{2}$ Division of Pulmonary and Critical Care \\ Medicine, New York University School of Medicine, NY 10016, USA and ${ }^{3}$ Department of Pathology, Teikyo University School of Medicine, Tokyo \\ 173-8605, Japan \\ Email: Harutaka Katano* - katano@nih.go.jp \\ * Corresponding author $\ddagger$ Presenting author
}

from 2005 International Meeting of The Institute of Human Virology Baltimore, USA, 29 August - 2 September 2005

Published: 8 December 2005

Retrovirology 2005, 2(Suppl I):S53 doi:I0.I I86/I742-4690-2-SI-S53

B cell lymphomas remain a significant cause of morbidity in AIDS but the pathophysiology of this disease is unclear. We report a case of B cell lymphoma in which HIV-1 integrated into the host genome. The lymphoma cells with anaplastic large cell morphology formed multiple nodular lesions in the lung of a homosexual AIDS patient. The lymphoma cells did not express KSHV-LANA and EBVEBER or HIV-1 p24 but did express high levels of nuclear localized STAT3. The provirus had a 5'LTR deletion and the 3'LTR was inserted just before the first coding exon of STAT3. Reporter gene assay demonstrated that the 3'LTR had a strong promoter activity especially when co-transfected with HIV Tat. These data suggest HIV-1 integration resulted in induction of STAT3 and possibly promoted lymphoma formation. This suggests that HIV-1 insertional mutagenesis may be associated with some cases of AIDS lymphoma. 